\title{
Nile red as a probe for lipid-storage products in benthic copepods
}

\author{
Kevin R. Carman ${ }^{1}$, David Thistle ${ }^{2}$, Stephen C. Ertman ${ }^{2}$, Michael Foy ${ }^{2}$ \\ ${ }^{1}$ Department of Zoology and Physiology, Louisiana State University, Baton Rouge, Louisiana 70803-1725, USA \\ ${ }^{2}$ Department of Oceanography, Florida State University, Tallahassee, Florida 32306-3048, USA
}

\begin{abstract}
Nile red (NR) is a hydrophobic fluorophore that binds specifically to neutral lipids. We examined the usefulness of NR as a probe for detecting neutral-lipid-storage products in benthic (harpacticoid) copepods. Following a brief (30 min) exposure to NR, lipid droplets, which fluoresce a bright yellow/orange, were clearly visible under epifluorescent illumination. Analysis of fluorescent images was used to determine relative lipid concentrations. The NR procedure was used on copepods subjected to a range of starvation periods. As predicted, detectable lipid reserves decreased with increased starvation period. The sensitivity of the NR procedure makes it a potentially useful tool for comparing relative lipid levels in individual organisms, even where lipid concentrations are near or below the detection limits of conventional analytical methods.
\end{abstract}

A variety of aquatic (marine and freshwater) organisms are known to use neutral lipids (e.g. triacylglycerols and wax esters) as carbon and energy reserves (Benson \& Lee 1975). Recently, there has been a proliferation of studies concerning the role of neutral lipids in the life histories of zooplanktonic crustaceans. It has been shown, for example, that neutral-lipid-storage products are important in their development and reproduction (Ohman 1987), and that levels of neutral-lipidstorage products are sensitive indicators of nutritional status (Håkanson 1984, 1987). In contrast to zooplankton, relatively little is known about the importance of lipid-storage products to benthic invertebrates, but existing data suggest that benthic animals use lipidstorage products in a fashion similar to that of zooplankton (e.g. Gardner et al. 1985, Quigley et al. 1989)

Among benthic animals, however, the importance of lipid-storage products in the life histories of meiofaunal organisms is essentially unknown. This information gap is, at least in part, due to technical difficulties associated with these small $(<1 \mathrm{~mm})$ invertebrates. For example, most benthic copepods have a dry weight of 1 to $10 \mu \mathrm{g}$. The amount of lipid that one would expect to recover from individual meiofaunal animals is near the detection limit of conventional analytical procedures (e.g. Iatroscan, gas chromatography, gravimetric analysis, high-performance liquid chromatography). Gardner et al. (1985) developed a micromethod for extracting lipids from small invertebrates, but it is quite timeconsuming. In spite of these technical limitations or difficulties, we are interested in using neutral lipids as indicators of the nutritional status of meiobenthic copepods (primarily harpacticoids). As an alternative to chemical-extraction techniques, we report here the use of nile red (NR) as a probe for determining relative lipid concentrations in individual copepods.

Nile red is a hydrophobic fluorophore that binds specifically to neutral lipids (Fowler \& Greenspan 1985, Cooksey et al. 1987). Nile red has recently been used to detect lipid-storage material in single-celled eukaryotes, including microalgae (Cooksey et al. 1987, Priscu et al. 1990) and ciliated protozoans (Cole et al. 1990). When viewed under epifluorescent illumination, neutral-lipid droplets in copepods stained with NR fluoresce a bright yellow/orange. Fluorescent images can be photographed or captured directly with imageanalysis software and subsequently analyzed to obtain a measure of the relative amount of lipid in an organism.

Methods. A stock solution of NR (Kodak, Rochester, $\mathrm{NY}$, USA) was prepared by adding $2.5 \mathrm{mg}$ of $\mathrm{NR}$ to $100 \mathrm{ml}$ of acetone. The solution was kept in an ambercolored bottle and stored in the dark at room temperature. Copepods were stained by placing them in a Petri dish containing $5 \mathrm{ml}$ of filtered seawater, then adding $200 \mu \mathrm{l}$ of NR stock solution to the dish. Staining of neutral lipids was complete within $30 \mathrm{~min}$. No destaining or rinses were required.

After staining, individual copepods were transferred to a depression slide in a drop of water (the staining solution in the Petri dish can be used because NR that is not bound to lipids will not fluoresce), and a cover slip 
was added. Copepods were examined at $100 \times$ using a Nikon Microphot FXA microscope and epifluorescent illumination ( $200 \mathrm{~W}$ mercury bulb). A standard FITC cube was used to set excitation (450 to $490 \mathrm{~nm}$ ) and emission (>510 nm) windows. Lipid droplets within copepods fluoresced a bright yellow/orange (Fig. 1).

A preliminary experiment was performed to determine if NR could be used to detect the expected decrease in lipid reserves that would result if copepods were starved. Robertsonia sp. were collected from an intertidal site near the Florida State University Marine Laboratory. Adult males were placed in Petri dishes (1 copepod per dish) that contained $5 \mathrm{ml}$ of filtered $(0.45$ $\mu \mathrm{m}$ ) seawater. Copepods were 'starved' under these conditions for $0,1,4$, and $6 \mathrm{~d}$. At the end of a starvation period, $200 \mu \mathrm{l}$ of NR stock solution were added to the dish, and copepods were stained for $30 \mathrm{~min}$. Ten individuals were analyzed for each starvation period. Individual copepods were examined under epifluorescent illumination, and color-slide micrographs (Ektachrome 400) of each copepod were taken. Each slide was mounted in a photographic enlarger, and the image of the copepod was projected from a standard height. The lipid droplets were then traced, the traced areas black-
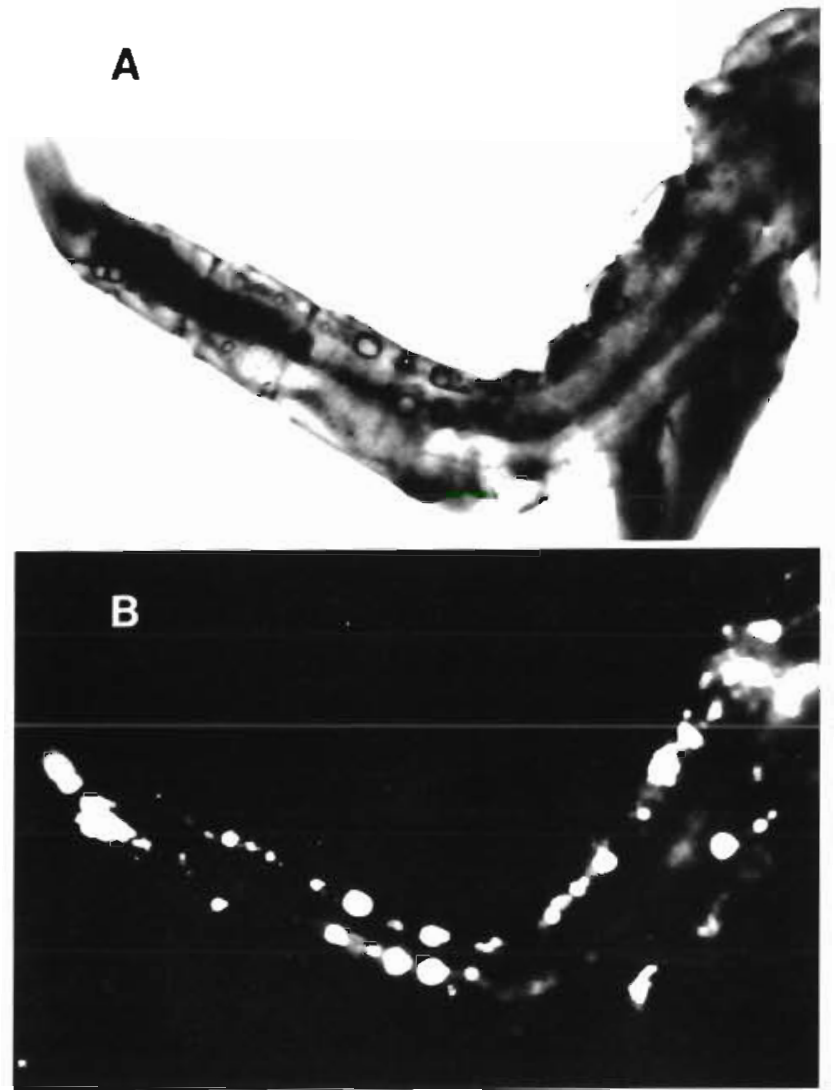

Fig. 1 Example of lipid deposits in Scottolana canadensis (A) as viewed under brightfield illumination, and (B) as viewed under epifluorescent illumination after staining with nile red. Lipid deposits are the bright fluorescent objects ened, and the blackened areas quantified with an Oculus 200 image-analysis system (Coreco, Montreal).

In a second experiment, we used a more automated approach to determine the depletion of copepod lipid reserves during a period of starvation. This experiment was similar to the first, except that adult females of Scottolana canadensis (collected from saltmarsh sediments near Louisiana Universities Marine Consortium - LUMCON, Cocodrie, LA) were used, and starvation periods were $0,1,3$, and $5 \mathrm{~d}$. For each starvation period, 20 individuals were examined. Images of copepods stained with NR were obtained with a Hamamatsu C2400 Chalnicon video camera attached to the microscope. Images were enhanced and saved using the Image-1/AT image-analysis system (Universal Imaging Corp., Ithaca, New York). Image analysis was performed using Micromeasure image-analysis software (Analytical Imaging Concepts, Irvine, California). An example of a brightfield and a fluorescent image of a specimen is given in Fig. 1. The area of lipid deposits was determined by adjusting the grey-scale threshold to optimize detection of fluorescing lipids. The optimal grey-scale setting was determined empirically, but to eliminate operator bias, all images were examined using the same threshold setting. To minimize problems with depth-of-field, separate determinations of lipid deposits in the urosome and prosome of each copepod were made.

A simple experiment was performed to compare the utility of quick-frozen vs formaldehyde-preserved samples for subsequent analysis using the NR procedure. Fresh sediments from the LUMCON saltmarsh were (1) preserved in $2 \%$ buffered formaldehyde, (2) frozen with liquid nitrogen and stored at $-50{ }^{\circ} \mathrm{C}$, or (3) placed on ice and transported immediately to the laboratory at Louisiana State University (a live-sampling technique). Live, adult females of Scottolana canadensis collected by the live-sampling technique were stained with NR and subjected to image analysis on the day of collection as described above. $S$. canadensis from formaldehyde-preserved samples were analyzed after $3 \mathrm{wk}$. S. canadensis preserved by freezing were analyzed after $3 \mathrm{wk}$ and $6 \mathrm{mo}$.

Finally, we examined the influence of Rose Bengal on the efficacy of the NR procedure. Rose Bengal is a vital stain that is commonly used as an aid in sorting of benthic samples. Of particular concern is that Rose Bengal may bind to lipids, and has fluorescent properties (Pearse 1960). Cores of sediment were collected from the Florida State University Marine Laboratory and quick-frozen with liquid nitrogen. Samples were thawed in the laboratory and copepods were concentrated by swirling and decanting (elutriation) onto a 62 um sieve. Adult males of Robertsonia sp. were examined under a compound microscope (256x) and separated 
into 'high-lipid' and 'low-lipid' groups based on the apparent abundance of lipid droplets in each copepod. Individuals with no apparent lipid droplets were discarded. From each group, half the copepods (selected at random) were placed in Petri dishes containing $4 \mathrm{ml}$ of autoclaved, $0.45 \mu \mathrm{m}$ filtered seawater (FSW), and $1 \mathrm{ml}$ of Rose Bengal stock solution $10.50 \mathrm{~g}$ Rose Bengal in 11 FSW) was added. The other half were placed in Petri dishes containing $4 \mathrm{ml}$ of FSW into which an additional $1 \mathrm{ml}$ of FSW was dispensed as a manipulation control. Both sets of Petri dishes were placed in a completely randomized pattern in an incubator and maintained at $18^{\circ} \mathrm{C}$ (approximately room temperature) for $20 \mathrm{~h}_{i}$ these conditions were chosen because meiobenthologists typically stain samples overnight at room temperature before sorting. We wanted to avoid the possible confounding influence of formaldehyde on the NR procedure, and thus autoclaved FSW was used to minimize the possibility of bacterial degradation of copepod lipids during the staining period. This treatment was apparently effective, as we saw no evidence that copepod lipid stores were diminished over the $20 \mathrm{~h}$ staining period. At the end of the incubation period, copepods
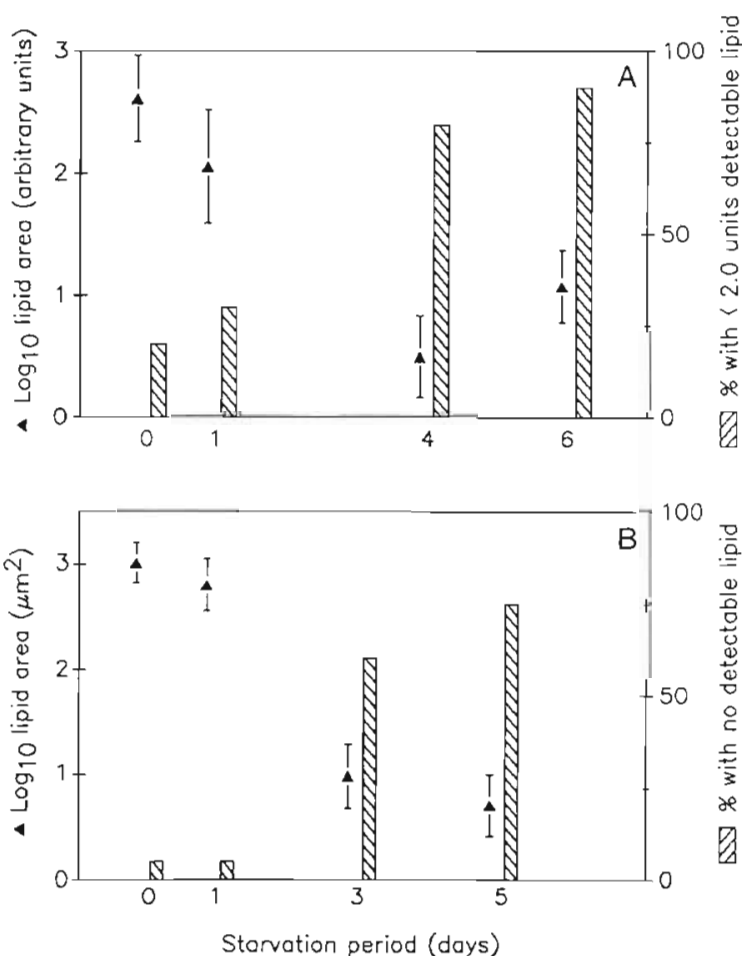

Fig. 2. Results of image analysis of lipid deposits in copepods subjected to a range of starvation periods. See 'Methods' for details of image-analysis procedures. (A) Area of lipid deposits (mean $\pm 1 \mathrm{SE}, \mathrm{n}=10$ ) in Robertsonia sp., and proportion of copepods with only trace quantities of lipids over a range of starvation periods. (B) Area of lipid deposits (mean $\pm 1 \mathrm{SE}, \mathrm{n}=20$ ) in the urosome of Scottolana canadensis, and proportion of copepods with no detectable lipids over a range of starvation periods were transferred to $5 \mathrm{ml}$ of FSW in Petri dishes and stained with NR as described above. Images of copepods were photographed (Ektachrome 400), and lipid area was quantified as described for the Robertsonia sp. starvation experiment.

Results and discussion. In the Robertsonia sp. starvation experiment, a modest depletion in lipid reserves occurred after $1 \mathrm{~d}$ of starvation, but after $4 \mathrm{~d}$ average lipid area decreased by a factor of 10 to 100 (Fig. 2A). Further, the proportion of copepods that contained only trace quantities of lipid reserves increased sharply after 4 or more days of starvation (Fig. 2A). These preliminary observations provided encouraging evidence that NR could be used to detect changes in levels of lipidstorage products in benthic copepods.

Results of the starvation experiment with Scottolana canadensis (Fig. 2B) were similar to those obtained in the experiment with Robertsonia sp. (Fig. 2A). A slight decrease in lipid reserves of $S$. canadensis was detected after $1 \mathrm{~d}$, and the urosomal area occupied by lipid reserves decreased by 2 orders of magnitude after $3 \mathrm{~d}$ of starvation. The proportion of copepods that had no detectable lipids increased after 3 and $5 \mathrm{~d}$, e.g. after $5 \mathrm{~d}$ of starvation, $75 \%(15 / 20)$ of the copepods had no detectable lipid reserves (Fig. 2B).

While the above experiments were performed on live specimens in the laboratory, the utility of the NR technique would be greatly enhanced if it could be used on preserved samples. Formaldehyde is commonly used as a fixative for field samples. We observed that brief $(\leq 24 \mathrm{~h}$ ) exposure to formaldehyde did not interfere with the NR staining procedure. When copepods were left in formaldehyde for $3 \mathrm{wk}$, detection of lipid deposits was not possible due to the presence of a bright red background fluorescence.

As an alternative to chemical fixation, quick-freezing in liquid nitrogen is a means of preserving the

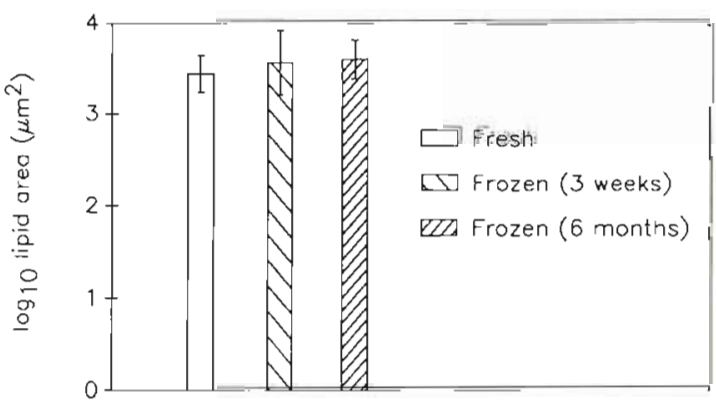

Fig. 3. Comparison of the effects of different preservation methods on the efficacy of the nile red technique for staining lipids. Preservation of Scottolana canadensis by freezing (liquid nitrogen, then stored at $-50^{\circ} \mathrm{C}$ ) and by fixation in $2 \%$ buffered formaldehyde was evaluated. Image analysis on copepods preserved in formaldehyde was impossible due to high background fluorescence. Data indicate area (mean \pm 1 $\mathrm{SE}, \mathrm{n}=10$ ) of lipids in the urosome of $S$. canadensis (as determined by image analysis) 
Table 1. Experiment to determine the influence of Rose Ben gal on the detection of lipid deposits using the nile red procedure. Adult males of Robertsonia sp. were separated into 'high-lipid' and 'low-lipid' groups. Half the copepods from each group were prestained with Rose Bengal (= Stained) and half were not (= Control). Values represent mean ( $\pm 1 \mathrm{SD}$ ) lipid area (arbitrary units), $n=10$

\begin{tabular}{|ccc|} 
& High-lipid & Low-lipid \\
\hline Stained & $6.38(4.86)$ & $1.20(1.20)$ \\
Control & $9.47(4.86)$ & $1.62(0.92)$ \\
\hline
\end{tabular}

biochemical integrity of field-collected samples (Dobbs \& LaRock 1989). In contrast to formaldehyde-preserved specimens, lipid deposits in quick-frozen copepods were readily detectable following $3 \mathrm{wk}$ at $-50^{\circ} \mathrm{C}$. The area occupied by lipid deposits in frozen specimens was not significantly different from that measured in fresh copepods [ $t$-test, $\mathrm{n}=10$ (fresh copepods), $\mathrm{m}=9$ (frozen copepods), $p=0.341$ ] (Fig. 3). Even after 6 mo of storage at $-50^{\circ} \mathrm{C}$, the detectable amount of lipids in Scottolana canadensis did not differ from that in freshly collected specimens ( $t$-test, $\mathrm{n}=10, \mathrm{~m}=9, \mathrm{p}=0.098$ ) (Fig. 3). It should be noted, however, that copepods kept in the freezer for 6 mo were fragile, apparently as a consequence of freezer burn.

As Rose Bengal is typically used as an aid to sorting benthic copepods (and other fauna), we felt it appropriate to determine if Rose Bengal staining interfered with the NR procedure. Detection of lipids in Robertsonia sp. was not significantly influenced by staining with Rose Bengal (Table 1; F-test, $0.05<\mathrm{p}<0.10$ ). It should be noted, however, that the average amount of detected lipid area was ca 1/3 less in both high-and low-lipid copepods when stained with Rose Bengal (Table 1). Thus, our experience indicates that prestaining copepods with Rose Bengal may somewhat reduce the sensitivity of the NR procedure, but the effect is independent of the amount of lipid present in the organism. It may therefore be acceptable to prestain specimens with Rose Bengal if one is concerned only with determining relative lipid concentrations among experimental treatments, for example.

Our observations indicate that NR is a sensitive probe for examining lipid deposits in individual benthic copepods. The level of lipid reserves, however, varied considerably even among individuals of the same age class and sex (Fig. 2, Table 1). The reason for this degree of natural variability in lipid reserves among cooccurring copepods is unknown, but should be kept in mind when determining the number of animals to be examined. Nevertheless, the technique is easy to use and allows detection of lipid quantities that are near or below the detection limit of conventional analytical procedures. We have observed that NR can be used to stain neutral lipids in a variety of crustaceans (e.g. calanoids, isopods, tanaids, amphipods) as well as nematodes. Thus, NR may be useful for examining lipid reserves in a variety of aquatic invertebrates. The bright fluorescence of neutral-lipid deposits allows the use of automated image analysis for determining relative amounts of lipid stores. This feature eliminates the requirement for manually measuring individual lipid droplets (Arts \& Evans 1991), and could reduce the subjectivity associated with the 'lipid index' used to determine the nutritional status of freshwater cladocerans (Tessier \& Goulden 1982).

Direct measurement of fluorescence of NR-stained lipids (with a fluorometer) has been used successfully on cultures of Chlorella sp. (Cooksey et al. 1987) and protozoans (Cole et al. 1990), and on natural assemblages of microalgae (Priscu et al. 1990). Although we have not yet attempted to do so, direct measurement of fluorescence by NR-stained lipids may represent an alternative and possibly more efficient means of quantification of lipid stores in copepods and other invertebrates.

Acknowledgements. J. Guckert suggested that we try nile red to examine lipid deposits in copepods. The manuscript was improved by comments from $F$. Dobbs and an anonymous reviewer. J. Lynn and B. Demler provided valuable instruction and assistance with the image analysis. Image analysis of copepods at Louisiana State University was performed using the facilities of the Life Sciences Microscopy Center of Louisiana State University, Baton Rouge. D. Balkwill (Florida State University) loaned his epifluorescence microscope and automatic camera. This research is contribution no. 1061 of the Florida State University Marine Laboratory. The research was supported, in part, by NSF grant OCE-8911181 (D.T.).

\section{LITERATURE CITED}

Arts, M. T., Evans, S. (1991). Optical-digital measurement of energy reserves in calanoid copepods: intersegmental distribution and seasonal patterns. Limnol. Oceanogr 36: 289-298

Benson, A. A., Lee, R. F. (1975). The role of wax in oceanic food chains. Scient. Amer 232: 76-86

Cole, T A., Fok, A. K., Ueno, M. S., Allen, R. D. (1990). Use of nile red as a rapid measure of lipid content in ciliates. Eur J. Protistol. 25: 361-368

Cooksey, K. E., Guckert, J. B., Williams, S. A., Callis, P. R (1987). Fluorometric determination of the neutral lipid content of microalgal cells using nile red. J, microbiol. Meth. 6 : 333-345

Dobbs, F. C., LaRock, P. A. (1989). The freezing of marine sediment for subsequent extraction of adenosine triphosphate. J. microbiol. Meth. 10: 113-117

Fowler, S. D., Greenspan, P. (1985). Application of nile red, a fluorescent hydrophobic probe, for the detection of neutral lipid deposits in tissue sections: comparison with oil red $O$. J. Histochem. Cytochem. 33: 833-836

Gardner, W. S., Frez, W. A., Cichocki, E. A., Parrish, C. C. (1985) Micromethod for lipids in aquatic invertebrates. Limnol. Oceanogr. 30: 1099-1105 
Hăkanson, J. L. (1984). The long and short term feeding condition in field-caught Calanus pacificus, as determined from the lipid content. Limnol. Oceanogr 29: $794-804$

Hảkanson, J. L. (1987). The feeding condition of Calanus pacificus and other zooplankton in relation to phytoplankton pigments in the Californa Current. Limnol. Oceanogr. 32: $881-894$

Ohman, M. D. (1987). Energy sources for recruitment of the subantarctic copepod Neocalanus tonsus. Limnol. Oceanogr 32: 1317-1330

This note was submitted to the editor
Pearse, A. G. E. (1960). Histochemistry, 2nd edn. Little, Brown $\&$ Co., Boston

Priscu, J. C., Priscu, L. R., Palmisano, A. C., Sullivan, C. W (1990). Estimation of neutral lipid levels in Antarctic sod ice microalgae by nile red fluorescence. Antarctic sci. 2: $149-155$

Quigley, M. A., Chandler, J. F., Gardner, W. S. (1989). Lipıd composition related to size and maturity of the amphipod Pontoporeia hoy. J. Great Lakes Res. 15: 601--610

Tessier, A. J., Goulden, C. E. (1982). Estimating food limitation in cladoceran populations. Limnol. Oceanogr 27: 707-717

Manuscript first received: January 8, 1991

Revised version accepted: May 24, 1991 